\title{
Dip-Pen Nanolithography Method for Fabrication of Biofunctionalized Magnetic Nanodiscs Applied in Medicine
}

\author{
(C) T.E. Smolyarova ${ }^{1,2}$, A.V. Lukyanenko ${ }^{1,2}$, A.S. Tarasov ${ }^{1,2}$, A.E. Sokolov ${ }^{1,2}$ \\ ${ }^{1}$ Siberian Federal University, \\ 660041 Krasnoyarsk, Russia \\ ${ }^{2}$ Kirensky Institute of Physics, Siberian Branch of the Russian Academy of Sciences, \\ 660036 Krasnoyarsk, Russia \\ E-mail: smol_nano@iph.krasn.ru
}

The magnetic properties of ferromagnetic nanodiscs coated with gold, manufactured using the Dip-Pen Nanolithography method, and were studied by atomic-force and magnetic force microscopy methods. The magnetic discs (dots) are represented as nanoagents (nanorobots) applied in medicine for the cancer cell destruction. The motivation of this work stem from the necessity of the understanding of the magnetization distribution in ferromagnetic discs that is crucial for their application in biomedicine. We have performed the theoretical calculations in order to compare the theoretical image contrast to experimental results. Herein, we report about the fabrication and analysis of biocompatible ferromagnetic nanodiscs with the homogenous magnetized state.

\section{Acknowledgment}

The study was funded by Russian Foundation for Basic Research, Government of Krasnoyarsk Territory, Krasnoyarsk Region Science and Technology Support Fund to the research project N 17-42-240080, N 16-42-243046, N 16-42-242036 and the Grant of the President of the Russian Federation No NSh-7559.2016.2. 